\title{
THE EFFECT OF CORRELATED VARIABILITY ON THE ACCURACY OF A POPULATION CODE
}

\author{
L. F. Abbott \\ Volen Center and Department of Biology \\ Brandeis University \\ Waltham MA 02254-9110 \\ and \\ Peter Dayan \\ Department of Brain and Cognitive Sciences \\ Massachusetts Institute of Technology \\ Cambridge, MA 02139
}

\begin{abstract}
We study the impact of correlated neuronal firing rate variability on the accuracy with which an encoded quantity can be extracted from a population of neurons. Contrary to a widespread belief, correlations in the variabilities of neuronal firing rates do not, in general, limit the increase in coding accuracy provided by using large populations of encoding neurons. Furthermore, in some cases, but not all, correlations improve the accuracy of a population code.
\end{abstract}




\section{$\underline{\text { Introduction }}$}

In population coding schemes, the activities of a number of neurons jointly encode the value of a quantity. A frequently touted advantage of population coding is that it suppresses the effects of neuronal variability. The observation of correlations in the trial-to-trial fluctuations of simultaneously recorded neurons (Gawne \& Richmond, 1993; Zohary et al, 1994; Lee et al, 1998) has raised some doubt as to whether this advantage is actually realized in real nervous systems. The dramatic effects of correlated variability can be seen by examining its impact on the average of $N$ neuronal firing rates. When the fluctuations of individual neurons about their mean rates are uncorrelated, the variance of the average decreases like $1 / N$ for large $N$. In contrast, correlated fluctuations cause the variance of the average to approach a fixed limit as the number of neurons increases. While illustrative, this example is not conclusive because the value of an encoded quantity can be extracted from a population of neurons by methods that do not require averaging their firing rates. Statements in the literature suggest that correlated variability can either decrease or increase (Snippe \& Koenderink, 1992; Shadlen \& Newsome, 1994; Shadlen et al, 1996; Reike et al., 1996; Oram et al., 1997; Lee et al, 1998) the accuracy of a population code. The purpose of this paper is to clarify this situation by addressing two questions: 1) Does correlation necessarily increase or decrease the accuracy with which the value of an encoded quantity can be extracted from a population of $N$ neurons? 2) Does this accuracy approach a fixed limit as $N$ increases?

This issue of correlated variability was first addressed by Johnson (1980b), who discussed circumstances under which correlation is either helpful or harmful for discrimination. Snippe and Koenderink (1992) studied the effect of correlated variability on optimal linear discrimination and also found some cases in which correlation improved discrimination and others where discrimination was degraded by correlation. We will study the effects of correlation on population coding accuracy by computing the Fisher information (Cox \& Hinckley, 1974; Paradiso, 1988; Seung \& Sompolinsky, 1993). The inverse of the Fisher information is the minimum averaged squared error for any unbiased estimator of an encoded variable. It thus sets a limit on the accuracy with which a population code can be read out by an unbiased decoding method.

Two simple examples illustrate the subtleties involved in analyzing the effects of correlation. Consider a set of $N$ neurons with firing rates $r_{i}$, for $i=1,2, \ldots N$, which have mean values $f_{i}$, identical variances $\sigma^{2}$, and correlated variabilities so that

$$
\left\langle\left(r_{i}-f_{i}\right)\left(r_{j}-f_{j}\right)\right\rangle=\sigma^{2}\left[\delta_{i j}+c\left(1-\delta_{i j}\right)\right]
$$

with correlation coefficient $c$ satisfying $0 \leq c<1$. The angle brackets on the left side of this equation denote an average over trials, and $\delta_{i i}=1$ for all $i$ while $\delta_{i j}=0$ 
if $i \neq j$. In this case, the variance of the average of the rates

$$
\bar{R}=\frac{1}{N} \sum_{i=1}^{N} r_{i}
$$

is

$$
\sigma_{\bar{R}}^{2}=\frac{\sigma^{2}}{N}[1+c(N-1)] .
$$

This illustrates two negative effects of correlation. For fixed $N$, the variance increases as a function of the degree of correlation $c$, and beyond $N \approx 1 / c$ the variance approaches a fixed limit $\sigma_{\bar{R}}^{2} \rightarrow c \sigma^{2}$, as discussed in the opening paragraph. Correlation among the activities of neurons in area MT of monkeys viewing moving random dot displays has been estimated at about $c=0.1-0.2$ (Zohary et al, 1994; Shadlen et al, 1996). This has led to the suggestion that coding accuracy will not improve for populations of more than about 100 neurons (Shadlen \& Newsome, 1994).

The second example may seem a bit contrived but is nevertheless illustrative. Consider the sign-alternating sum

$$
\tilde{R}=\frac{1}{N} \sum_{i=1}^{N}(-1)^{i} r_{i} .
$$

The variance of this quantity (for even $N$ ) is

$$
\sigma_{\tilde{R}}^{2}=\frac{\sigma^{2}}{N}(1-c)
$$

For this variable, positive correlation always decreases the variance, and the variance is proportional to $1 / N$ whether or not correlation is present.

One reason to think that correlation need not always be harmful is that it generally reduces the entropy of the variability in a neural population, suggesting that it should therefore increase the accuracy of a population code. Our results on population coding generally concur with this entropy analysis. For the cases we consider, the lower limit on the averaged squared decoding error provided by the Fisher information is proportional to $1 / N$ for large $N$, similar to the behavior of equation (5) not (3). For additive or multiplicative noise with uniform correlations, the dependence on the degree of correlation $c$ also resembles that of equation (5), and thus correlation improves population coding accuracy. We also consider correlations of limited range for which coding accuracy can display both increasing and decreasing behavior (Snippe \& Koenderink, 1992).

\section{The Model}

We consider a population code in which $N$ neurons respond to a stimulus with firing rates that depend on a variable $x$ that parameterizes some stimulus attribute 
(Johnson, 1980a\&b; Georgopoulos, Schwartz \& Kettner, 1986; Paradiso, 1988; Baldi \& Heiligenberg, 1988; Snippe \& Koenderink, 1992; Seung \& Sompolinsky, 1993; Salinas \& Abbott, 1994; Snippe, 1996; Sanger, 1996). The activity of neuron $i$, averaged over trials that use the stimulus $x$, is $f_{i}(x)$, and its activity on any given trial is

$$
r_{i}=f_{i}(x)+\eta_{i} .
$$

We interpret this as the number of spikes fired by the neuron over a fixed time period. We do not discuss encoding that involves the fine-scale temporal structure of spike trains. The random terms $\eta_{i}$ for $i=1,2, \ldots, N$ are generated from a Gaussian probability distribution with zero mean and covariance matrix $\mathbf{Q}(x)$. We consider three different models of variability. In the additive noise model (Johnson, $1980 b$ ), the covariance matrix is identical to (1),

$$
Q_{i j}=\sigma^{2}\left[\delta_{i j}+c\left(1-\delta_{i j}\right)\right] .
$$

For multiplicative noise, the variability in the firing rate is still described by Equation (6), but the covariance matrix is scaled by the average firing rates,

$$
Q_{i j}(x)=\sigma^{2}\left[\delta_{i j}+c\left(1-\delta_{i j}\right)\right] f_{i}(x) f_{j}(x) .
$$

This produces variances that increase as a function of firing rate, and larger correlations for neurons with overlapping tuning curves, as is seen in the data (Lee et al, 1998). We also consider a model in which the correlations can have an even more dramatic dependence on the distance between tuning curves. This is the limited range correlation model (Snippe \& Koenderink, 1992) with the correlation matrix written as

$$
Q_{i j}=\sigma^{2} \rho^{|i-j|}
$$

where the parameter $\rho$ (with $0<\rho<1$ ) determines the range of the correlations between different neurons in the population. The parameter $\rho$ can be expressed in terms of a correlation length $L$ by writing

$$
\rho=\exp (-\Delta / L)
$$

where $\Delta$ is the spacing between the peaks of adjacent tuning curves.

We use the notation $\mathbf{Q}$ to denote the matrix with elements $Q_{i j}$, and $\mathbf{r}$ and $\mathbf{f}(x)$ to denote the vectors of firing rates with elements $r_{i}$ and $f_{i}(x)$ respectively. Note that in the additive and limited range cases, $\mathbf{Q}$ does not depend on $x$, while for multiplicative noise it does.

The average firing rates $\mathbf{f}(x)$ are the tuning curves of the neurons in the population. We imagine the the tuning curves are arranged to cover a range of $x$ values, with different tuning curves localized to different ranges of $x$. We assume that the coverage is dense and roughly uniform (we define these terms below), but otherwise leave the exact nature of these tuning curves relatively unrestricted. 


\section{Fisher Information}

The Fisher information provides a useful measure of the accuracy of a population code. Through the Cramér-Rao bound, the Fisher information limits the accuracy with which any unbiased decoding scheme can extract an estimate of an encoded quantity from the activity of a population of neurons. The average value of an unbiased estimate is equal to the true value, $x$, of the encoded quantity, but the estimate will typically differ from $x$ on a trial-to-trial basis. For an unbiased estimate, the average squared decoding error is equal to the variance of these trial-to-trial deviations. The Cramér-Rao bound states that the average squared decoding error for an unbiased estimate is greater than or equal to $1 / I_{F}(x)$, where $I_{F}(x)$ is the Fisher information. Although, in some cases, an biased decoding scheme may outperform a biased method, no biased estimate can do better than the Cramér-Rao lower bound. To compute the Fisher information, we need to know the conditional probability distribution $P[\mathbf{r} \mid x]$ which determines the probability that a given response $\mathbf{r}$ is evoked by the stimulus $x$. The Fisher information is given in terms of $P[\mathbf{r} \mid x]$ by

$$
I_{F}(x)=-\int d \mathbf{r} P[\mathbf{r} \mid x] \frac{d^{2} \log P[\mathbf{r} \mid x]}{d x^{2}} .
$$

The maximum likelihood estimator, which chooses for an estimate the value of $x$ that maximizes $P[\mathbf{r} \mid x]$, asymptotically saturates the Cramér-Rao bound as $N \rightarrow$ $\infty$. Thus, the bound sets a realizable limit making it a good measure of the accuracy of a population code (see Paradiso, 1988; Seung \& Sompolinsky, 1993; and Pouget et al, 1998 for discussions of the use of ML techniques and Fisher information for population codes in the absence of correlation). The psychophysical measure of discriminability $d^{\prime}$ that quantifies how accurately discriminations can be made between two slightly different values $x$ and $x+\Delta x$ based on $\mathbf{r}$ is related to the Fisher information by the formula

$$
d^{\prime}=\Delta x \sqrt{I_{F}(x)} .
$$

The larger the Fisher information, the better the discriminability and the smaller the minimum unbiased decoding error.

When the random $\eta$ terms are generated from a Gaussian probability distribution as discussed above,

$$
P[\mathbf{r} \mid x]=\frac{1}{\sqrt{(2 \pi)^{N} \operatorname{det} \mathbf{Q}(x)}} \exp \left[-\frac{1}{2}[\mathbf{r}-\mathbf{f}(x)]^{T} \mathbf{Q}^{-1}(x)[\mathbf{r}-\mathbf{f}(x)]\right] .
$$

This equation does not give zero probability for negative firing rates, but we assume that the means and variances are such that this has a small effect. Substituting (13) into Equation (11), we find (see, for example, Kay, 1993)

$$
I_{F}(x)=\mathbf{f}^{\prime}(x)^{T} \mathbf{Q}^{-1}(x) \mathbf{f}^{\prime}(x)+\frac{1}{2} \operatorname{Tr}\left[\mathbf{Q}^{\prime}(x) \mathbf{Q}^{-1}(x) \mathbf{Q}^{\prime}(x) \mathbf{Q}^{-1}(x)\right]
$$


where $\operatorname{Tr}$ stands for the trace operation,

$$
\mathbf{Q}^{\prime}(x)=\frac{d \mathbf{Q}(x)}{d x} \quad \text { and } \quad \mathbf{f}^{\prime}(x)=\frac{d \mathbf{f}(x)}{d x} .
$$

When $\mathbf{Q}$ is independent of $x$, as it is for additive noise and limited range correlations, this reduces to

$$
I_{F}(x)=\mathbf{f}^{\prime}(x)^{T} \mathbf{Q}^{-1} \mathbf{f}^{\prime}(x) .
$$

Equations (16) and (14) are the basis for all our results. To apply them we need the inverses of the covariance matrices which are, in the additive case

$$
\left[\mathbf{Q}^{-1}\right]_{i j}=\frac{\delta_{i j}(N c+1-c)-c}{\sigma^{2}(1-c)(N c+1-c)}
$$

in the multiplicative case,

$$
\left[\mathbf{Q}^{-1}(x)\right]_{i j}=\frac{\delta_{i j}(N c+1-c)-c}{f_{i}(x) f_{j}(x) \sigma^{2}(1-c)(N c+1-c)}
$$

and, in the case of correlations with limited range

$$
\left[\mathbf{Q}^{-1}\right]_{i j}=\frac{1+\rho^{2}}{\sigma^{2}\left(1-\rho^{2}\right)}\left[\delta_{i j}-\frac{\rho}{1+\rho^{2}}\left(\delta_{i+1, j}+\delta_{i-1, j}\right)\right] \text {. }
$$

\section{Results}

\subsection{Additive Noise}

The Fisher information in the additive case is computed by substituting the correlation matrix (7) into Equation (16) and doing the necessary algebra. The result depends on two sums,

$$
F_{1}(x)=\frac{1}{N} \sum_{i}\left(f_{i}^{\prime}(x)\right)^{2} \quad \text { and } \quad F_{2}(x)=\left(\frac{1}{N} \sum_{i} f_{i}^{\prime}(x)\right)^{2}
$$

These have been scaled to be of order one for the case of uniform tuning curve placement. In terms of these quantities,

$$
I_{F}(x)=\frac{c N^{2}\left[F_{1}(x)-F_{2}(x)\right]+(1-c) N F_{1}(x)}{\sigma^{2}(1-c)(N c+1-c)} .
$$

As $N$ tends to infinity,

$$
I_{F}(x) \rightarrow \frac{N\left[F_{1}(x)-F_{2}(x)\right]}{\sigma^{2}(1-c)}
$$


which grows with $N$ and $c$ provided that $F_{1}(x)-F_{2}(x)>0$. Note that, aside from the factor of $F_{1}(x)-F_{2}(x)$, the inverse of this expression matches the variance of Equation (5). For large $N$, a uniform array of tuning curves should generate functions $F_{1}(x)$ and $F_{2}(x)$ that are insensitive to the values of either $x$ or $N$ (indeed, this is our definition of uniform tuning curve placement). Tuning curve arrays that are symmetric with respect to the sign flip $x \rightarrow-x$ (that is, for every neuron with tuning curve $f(x)$ there is another neuron with tuning curve $f(-x)$ ) have $F_{2}(x)=0$.

In the additive noise case, the inverse of the Fisher information, which determines the minimum unbiased decoding error, decreases as $1 / N$ for large $N$, and also decreases as a function of $c$, the degree of correlation. The Fisher information diverges, and the minimal error goes to zero as $c$ approaches one. As $c \rightarrow 1$, any slight difference in the tuning curves can be exploited to calculate the noise exactly and remove it.

Zhang and Sejnowski (1998) have noted an interesting scaling property of the Fisher information that also appears in our results. They considered the simultaneous encoding of $D$ variables by a population of neurons, and studied the effect of changing tuning curve width on encoding accuracy. If the widths of the tuning curves of the encoding population are scaled by a parameter $w$, we expect $F_{1}$ to scale like $w^{D-2}$. The factor of $w^{D}$ reflects the number of responding neurons, while the factor $w^{-2}$ is due to the squared derivative. For simplicity, we take $F_{2}=0$. Then, the Fisher information satisfies $I_{F} \propto N w^{D-2} / \sigma^{2}$ in agreement with the results of Zhang and Sejnowski (1998).

The Fisher information we have computed increases as a function of $c$ and $N$ unless $F_{1}(x)-F_{2}(x)=0$ or $F_{1}(x)-F_{2}(x) \rightarrow 0$ for large $N$. By the CauchySchwartz inequality $F_{1}(x) \geq F_{2}(x)$. For large $N, F_{1}(x)-F_{2}(x)$ could go to zero if both $F_{1}(x) \rightarrow 0$ and $F_{2}(x) \rightarrow 0$. We define the tuning curve coverage as being dense if $F_{1}(x) \nrightarrow 0$ for any $x$, since this implies that as more neurons are added, a fixed fraction respond significantly to $x$. By the condition for equality in the Cauchy-Schwartz inequality, the other alternative, $F_{1}(x)=F_{2}(x)$, requires $f_{i}^{\prime}(x)$ to be independent of $i$ or, equivalently,

$$
f_{i}(x)=p(x)+q_{i}
$$

for any function $p$ and numbers $q_{i}$. Thus, the Fisher information will fail to grow as a function of $c$ and $N$ only if there is an additive separation of dependency between the value $x$ and the index $i$. This means that correlation is only harmful in the case where all the neurons share the same tuning dependence on $x$. This is not normally the case since neurons almost always have some variability in their stimulus preferences. Of course, we must assume that the mechanism that reads out the encoded quantity takes advantage of this variability and does not simply perform an averaging operation. 


\subsection{Multiplicative Noise}

When $\mathbf{Q}(x)$ is given by Equation (8), the Fisher information defined by Equation (14) depends on the logarithmic derivatives of the average firing rate tuning curves

$$
g_{i}^{\prime}(x)=\frac{1}{f_{i}(x)} \frac{d f_{i}(x)}{d x}=\frac{d \ln f_{i}(x)}{d x} .
$$

In particular, it depends on the sums

$$
G_{1}(x)=\frac{1}{N} \sum_{i}\left(g_{i}^{\prime}(x)\right)^{2} \quad \text { and } \quad G_{2}(x)=\left(\frac{1}{N} \sum_{i} g_{i}^{\prime}(x)\right)^{2}
$$

and is given by

$$
\begin{aligned}
I_{F}(x) & =\frac{c N^{2}\left[\left[G_{1}(x)-G_{2}(x)\right]+(1-c) N G_{1}\right.}{\sigma^{2}(1-c)(N c+1-c)} \\
& +\frac{\left[N^{2} c(2-c)+2 N(1-c)^{2}\right] G_{1}(x)-c^{2} N^{2} G_{2}(x)}{(1-c)(N c+1-c)} .
\end{aligned}
$$

For large $N$, this approaches the limit

$$
I_{F}(x) \rightarrow \frac{N\left[G_{1}(x)-G_{2}(x)\right]}{\sigma^{2}(1-c)}+\frac{N\left[(2-c) G_{1}(x)-c G_{2}(x)\right]}{(1-c)} .
$$

The Fisher information for multiplicative noise contains one term that depends on the noise variance $\sigma^{2}$, and another term that, surprisingly, is independent of $\sigma^{2}$. This second term arises because, with multiplicative noise, the encoded variable can be estimated from second-order quantities, not merely from measurements of the firing rates themselves.

The Fisher information of Equation (27) is proportional to $N$ and is an increasing function of $c$ provided that $G_{1}(x)>G_{2}(x)$. Since $G_{1}(x) \geq G_{2}(x)$ by the Cauchy-Schwartz inequality, the only way to modify this behavior is if $G_{1}(x)=$ $G_{2}(x)$. This condition only holds if $g_{i}^{\prime}(x)$ is independent of $i$ or, equivalently, if

$$
f_{i}(x)=p(x) q_{i}+r(x)+s_{i}
$$

for any functions $p$ and $r$ and numbers $q_{i}$ and $s_{i}$. This is multiplicative separability rather than the additive separability of Equation (23). As in the case of additive noise, the Fisher information with multiplicative noise increases with correlation and grows linearly with $N$ unless a contrived set of tuning curves is used.

\subsection{Limited Range Correlations}

In both of the cases we have studied thus far, the accuracy of the population code, quantified by the Fisher information, increase both as a function of $N$ and $c$. While 
the linear dependence of the Fisher information on $N$ appears quite general, there are cases in which introducing correlation decreases rather than increases $I_{F}$ (Johnson, 1980b; Snippe \& Koenderink, 1992). One example is provided by the limited range correlations described by the matrix of Equation (9). The Fisher information for this case is

$$
I_{F}(x)=\frac{N(1-\rho) F_{1}(x)}{\sigma^{2}(1+\rho)}+\frac{N^{1-2 / D} \rho F_{3}(x)}{\sigma^{2}\left(1-\rho^{2}\right)}
$$

where $F_{1}$ is as given above, $D$ is the number of encoded variables, and (provided that $x$ is away from the edges of the range covered by the population tuning curves)

$$
F_{3}(x)=N^{2 / D-1} \sum_{i=1}^{N}\left(f_{i+1}^{\prime}(x)-f_{i}^{\prime}(x)\right)^{2} .
$$

The power of $N$ in the definition $F_{3}(x)$ is chosen so that it is independent of $N$ for uniform tuning curve coverage. As $N$ gets large, the distance between neighboring tuning curves decreases as $N^{-1 / D}$, and the difference between their derivatives is proportional to this factor.

For a fixed value of $N$, the Fisher information in Equation (29) is a nonmonotonic function of the parameter $\rho$ that determines the range and degree of the correlations. The first term in Equation (29) is a decreasing function of $\rho$ and hence of $L$, the correlation length introduced in Equation (10), while the second term has the opposite dependence. For a fixed $N$ value, the first term dominates for small $L$, and the second dominates for large $L$.

For any finite value of $D$, the first term in Equation (29) will dominate for large $N$, so as $N \rightarrow \infty$

$$
I_{F}(x) \rightarrow \frac{N(1-\rho) F_{1}(x)}{\sigma^{2}(1+\rho)} .
$$

Note that this limit is approached more rapidly for small $D$ than for large $D$. The expression in (31) is a decreasing function of $\rho$, so in this case, unlike the additive and multiplicative cases, increasing correlation decreases the Fisher information. However, the Fisher information still grows linearly with $N$ for any $\rho<1$. The only way to prevent the Fisher information from growing with $N$ is to force $\rho$ nearer and nearer to 1 as $N \rightarrow \infty$. For example, if $\rho=c^{1 / N}$, for $0<c<1$, the Fisher information tends to a constant as $N \rightarrow \infty$.

\section{Discussion}

We have studied how correlations between the activities of neurons within a coding population affect the accuracy with which an encoded quantity can be determined or discriminated (Johnson, 1980b). We find that, generically, correlations between units do not prevent the Fisher information from growing linearly with the number 
of encoding neurons, and correlations can either improve or degrade decoding accuracy depending on the form of the correlation matrix. Only in the limit as the correlations get very close to 1 can this behavior change in some circumstances. Since our results are based on the Fisher information, they apply only to unbiased estimates. However, biased estimates would presumably be used only to further improve accuracy, and thus the increase in accuracy with $N$ would not be destroyed by using a biased estimate. Thus, optimal population-based estimates do not suffer from the limitations that correlation imposes on estimates of average firing rates. While averaging can be used to obtain more accurate firing-rate estimates from a group of neurons, it does not improve the accuracy of a population decoding procedure.

There are nevertheless possible lacunæ in our analysis. We only considered relatively simple noise models. We also used noise with Gaussian statistics. Poisson noise would be an obvious alternative and would entail slightly different calculations. Finally, we did not consider the computational complexity or biological implementation of the optimal decoding algorithms, although a good point of departure would be the work of Pouget et al (1998) showing how to perform ML inference using a recurrent network in the case without correlations.

The most relevant requirement for retaining the improved accuracy provided by large populations of encoding neurons is that the neurons should have different selectivities to the quantity they are jointly encoding. In particular, their tuning curves must not be additively or multiplicatively separable. Tuning functions that are commonly adopted in modeling work and seen in biological systems do not appear to have these problematic features.

\section{Acknowledgments}

We are grateful to Peter Latham, Alex Pouget, Sebastian Seung, Michael Shadlen and Haim Sompolinsky for discussions and comments. Research supported by the National Science Foundation (DMS-9503261) and the WM Keck Foundation for LFA, and by the National Institute of Mental Health (MH-55541) and the National Science Foundation (IBM-9634339) for PD.

\section{References}

Baldi, P \& Heiligenberg, W (1988) How sensory maps could enhance resolution through ordered arrangements of broadly tuned receivers. Biological Cybernetics 59:313-318.

Cox, DR \& Hinckley, DV (1974) Theoretical Statistics. London:Chapman \& Hall.

Foldiak, P (1993) The 'ideal homunculus': statistical inference from neural population responses. In Eeckman, FH \& Bower, J eds., Computation and Neural Systems, Norwell MA:Kluwer Academic, pp. 55-60. 
Georgopoulos, AP, Schwartz, AB \& Kettner, RE (1986) Neuronal population coding of movement direction. Science 243:1416-1419.

Gawne, TJ \& Richmond, BJ (1993) How independent are the messages carried by adjacent inferior temporal cortical neurons? Journal of Neuroscience 13:27582771.

Johnson, KO (1980a) Sensory discrimination: decision process. Journal of Neurophysiology 43:1771-1792.

Johnson, KO (1980b) Sensory discrimination: neural processes preceding discrimination decision. Journal of Neurophysiology 43:1793-1815.

Kay, SM (1993) Fundamentals of Statistical Signal Processing: Estimation Theory. Englewood Cliffs, NJ:Prentice-Hall.

Lee, D, Port, NL, Kruse, W \& Georgopoulos, AP (1998) Variability and correlated noise in the discharge of neurons in motor and parietal areas of the primate cortex. Journal of Neuroscience 18:1161-1170.

Oram, MW, Foldiak, P, Perett, DI \& Sengpiel, F (1997) The 'ideal homunculus': decoding neural population signals. Trends in Neuroscience (in press).

Paradiso, MA (1988) A theory for the use of visual orientation information which exploits the columnar structure of striate cortex. Biological Cybernetics 58:3549.

Pouget A, Zhang K, Deneve S \& Latham P (1998) Statistically Efficient Estimation Using Population Coding. Neural Computation 10:2-8.

Reike, F, Warland, D, de Ruyter van Steveninck, R \& Bialek, W (1996) Spikes: Exploring the Neural Code. Cambridge MA:MIT Press.

Salinas, E \& Abbott, LF (1994) Vector reconstruction from firing rates. Journal of Computational Neuroscience 1:89-107.

Sanger, TD (1996) Probability density estimation for the interpretation of neural population codes. Journal of Neurophysiology 76:2790-2793.

Seung, HS \& Sompolinsky, H (1993) Simple models for reading neuronal population codes. Proc. Natl. Acad. Sci. USA 90:10749-10753.

Shadlen, MN, Britten, KH, Newsome, WT \& Movshon, JA (1996) A computational analysis of the relationship between neuronal and behavioral responses to visual motion. Journal of Neuroscience 16:1486-1510.

Shadlen, MN \& Newsome, WT (1994) Noise, neural codes and cortical organization. Current Opinion in Neurobiology 4:569-579.

Snippe, HP (1996) Parameter extraction from population codes: a critical assessment. Neural Computation 8:511-530.

Snippe, HP \& Koenderink, JJ (1992) Information in channel-coded systems: correlated receivers. Biological Cybernetics 67:183-190. 
Zhang K \& Sejnowski T (1998) Neural tuning: to sharpen of broaden? Neural Computation (in press).

Zohary E, Shadlen MN, \& Newsome, WT (1994) Correlated neuronal discharge rate and its implications for psychophysical performance. Nature 370:140-143. 